appears either that there is an instrumental problem and the highest velocity gas is not real (or possibly, that calibration uncertainties have been underestimated), or that some of the molecular gas is concentrated in a galaxy-sized part, and another part, much more extended and of nearly equal mass, does not emit visible radiation.

Observations at IRAM by a largely European group suggest that the difference between the properties of F10214+4724 and other known galaxies may not be as extreme as was first thought. The dust mass ${ }^{5}$ is somewhat less than originally determined by Rowan-Robinson et al.; and neither is there as much $\mathrm{H}_{2}$ as others estimated ${ }^{6}$. The IRAM group concludes that F10214+4724 is similar to but at the extreme end of a class of ultraluminous galaxies identified from the IRAS infrared galaxy survey. These galaxies are as luminous as quasars, but like $\mathrm{F} 10214+4724$, emit most of their radiation in the infrared portion of the spectrum rather than in the optical range. Their luminosities are generally attributed to an enormous burst of star formation triggered by the merger of two spiral galaxies. The authors argue that F10214+4724 appears to be a more extreme example of these exotic, but better known galaxies.

But once again we run into observational and conceptual difficulties. The flux density in the $\mathrm{CO}$ line measured by the European investigators is considerably less than that measured by the Japanese team, even though their singledish observations measured a larger area than did the Nobeyama array. The opposite should have been observed. In any event, a fundamental conundrum appears in trying to fathom all the data. The lower mass estimate makes the narrow line widths more palatable, and does not require an unlikely viewing angle. On the other hand, the lower mass estimate is difficult to reconcile with the observed carbon abundance.

Neutral gaseous carbon was detected ${ }^{1}$ from F10214+4724 in two different transitions, which makes it possible to get a reasonably good estimate of the total mass of atomic carbon, about $1-3 \times 10^{8}$ solar masses. Carbon is believed to be a product of stellar nucleosynthesis, its presence in the interstellar medium signalling its manufacture in the centres of massive stars which then return it to the interstellar medium when the stars explode as supernovae. Near the Sun, the carbon abundance relative to hydrogen is $4 \times 10^{-3}$ by mass, but this has been built up over the age of the Universe. In $\mathrm{F} 10214+4724$, if the $\mathrm{H}_{2}$ mass is as small as $1 \times 10^{11}$ solar masses as suggested by the European and the Japanese investigators, then the carbon abundance would be nearly equal to the solar abundance. How is it possible for a galaxy five times younger than the Milky Way to have manufactured and dispersed such a disproportionate amount of carbon? If Brown and vanden Bout's $\mathrm{H}_{2}$ mass estimate of about $10^{12}$ masses is closer to the correct one, the carbon abundance is more palatable, but the discrepancy between the large mass and narrow line width is once more resurrected. Does it make sense that we observe a unique galaxy, which could be oriented in any direction, at a highly improbable angle? Or that a massive primaeval galaxy should be a thin disk?

As observers wrestle with these questions, other tantalizing questions and possibilities arise. For example, in normal galaxies, massive stars make up only a small fraction of the total stellar inventory of a galaxy. Does F10214+4724 have its normal complement of low-mass stars? According to standard stellar evolutionary theory, the large burst of massive-star formation needed to explain the infrared luminosity would leave enormous numbers of black hole remnants within a few million years of the starburst that we are currently observing. So, unless the galaxy is unique (an unlikely prospect) there may be a large number of galaxies with tens of millions of black holes in their midst.

In spite of the inconsistencies, it is important to recognize that the problems in deciphering the observations of F10214+4724 do not come from the shortcomings of the observers, but are largely the result of instruments being pushed to their limits. Notwithstanding all the difficulties, one thing is clear: F10214+4724 is an extraordinary galaxy in which a uniquely large fraction of its mass is in the form of molecular gas making massive stars at a prodigious rate. The observational evidence does suggest that it is a galaxy that is in an early evolutionary stage and F10214+4724 may thus be a Rosetta Stone for understanding how galaxies form. However, it will take much more work to decipher its hieroglyphics.

Leo Blitz is in the Astronomy Department, University of Maryland, College Park, Maryland 20742, USA.

\footnotetext{
1. Brown, R. L. \& vanden Bout, P. A. Astrophys. J. 397 L11-L14 (1992)

Brown, R. L. \& vanden Bout, P. A. Astrophys. J. 397 L19-L22 (1992)

3. Kawabe, R., Sakamoto, K., Ishizuki. S. \& Ishiguro, M. Astrophys. J. 397, L23-L26 (1992)

. Sakamoto, K., Ishizuki, S., Kawabe, R. \& Ishiguro, M Astrophys. J. 397, L27-L30 (1992)

5. Downes, D. et al. Astrophys. J. 398, L25-L28 (1992)

6. Solomon, P. M., Downes, D. \& Radford, S. J. E. Astrophys. J. 398, L.28- L32 (1992).

Rowan-Robinson, M. et al Nature 351, 719. 721 (1991).

8. Brown, R. L. \& vanden Bout, P. A. Astr. J. 102, 1956-1959 (1991).

9. Solomon, P. M., Radford, S. J. E. \& Downes, D. Nature
} 356. 318-319 (1992)

\section{Fulminating soot}

IN the past few years, the humble carbon arc has proved a prolific source of wonderful new carbon allotropes buckminsterfullerene, other fullerenes and carbon nanotubes. All these molecules assemble themselves from carbon evaporated from the electrodes. Now Ostwald's rule says that an element condenses from the vapour into its least stable allotropic form. Thus phosphorus vapour condenses to yellow phosphorus, not the more stable red variety. So Daedalus reckons that the vapour from a carbon arc should generate the most unstable carbon allotropes of them all. These are the carbynes, linear high polymers of carbon atoms linked by alternate single and acetylenic bonds, and usually too explosive to isolate. But intrepid DREADCO chemists are striking carbon arcs under liquefied noble gases, which should safely quench and contain the growing carbyne chains as they form.

To isolate the products safely, Daedalus will tip in a large excess of some inert polymer. The carbyne will be diluted to harmlessness in the resulting polymeric matrix. The mixed polymer could even be moulded and stretched to align the straight-chain carbyne molecules from end to end of the sample.

At first, Daedalus felt that the product would be a novel organic conductor. A carbyne molecule, as the ultimate fine carbon filament, should conduct electricity. But he then realized the disruptive consequences. Electricity is a flow of electrons. A flow of electrons along a carbyne molecule would shift all the bonding electrons one step along. The molecule would be disrupted into pairs of $C_{2}$ radicals in a single-molecule explosion. The radicals would probably then combine with their polymer matrix.

An explosive fired directly by electricity, and going to a solid product, should be very useful. It would react instantaneously throughout the currentcarrying volume: no shock-wave would be needed to carry the explosion from point to point. So a plastic containing very little carbyne, far too dilute to propagate a shock-wave and therefore quite safe against flame or impact, could be exploded electrically. It would give an equally dilute blast - simply a sudden expansion of the plastic block.

DREADCO's 'Carbynite' will transform engineering. It will be formed into all sorts of simple, reliable, pre-calibrated, electrically triggered actuators, releasers and jacks. It will split rock, shift jammed loads, drive nails, and operate emergency brakes and stopvalves. Wherever a controlled force needs to be applied once only, safely and certainly, Carbynite will be there. David Jones

NATURE · VOL 360 - 5 NOVEMBER 1992 